\title{
BOOSTING CED USING ROBUST ORIENTATION ESTIMATION
}

\author{
Tariq M. Khan ${ }^{2}$, Mohammad A. U. Khan ${ }^{1}$, Yinan Kong ${ }^{3}$ \\ ${ }^{1,3}$ Department of Engineering, Macquarie University, Sydney, Australia \\ ${ }^{2}$ Department of Electrical and Computer Engineering, \\ Effat University, Jeddah, Saudi Arabia.
}

\begin{abstract}
In this paper, Coherence Enhancement Diffusion (CED) is boosted feeding external orientation using new robust orientation estimation. In CED, proper scale selection is very important as the gradient vector at that scale reflects the orientation of local ridge. For this purpose a new scheme is proposed in which pre calculated orientation, by using local and integration scales. From the experiments it is found the proposed scheme is working much better in noisy environment as compared to the traditional Coherence Enhancement Diffusion
\end{abstract}

\section{KEYWORDS}

Fingerprint Image Enhancement, Coherence Enhancement Diffusion Directional Filter Bank (DFB), Decimation free Directional Filter Bank (DDFB).

\section{INTRODUCTION}

Biometric is a science that deals with the identification of human by using their traits or characteristics. It is based on measurable and distinctive characteristics which are used to label and describe individuals [1]. Examples include, face recognition, DNA, palm print, hand geometry, retina, iris recognition and finger- print. Fingerprint identification is one of the oldest biometric techniques which have been successfully used in numerous applications [2]. Every person has a unique and immutable fingerprint. A fingerprint surface contains series of ridges and valleys/furrows. These patterns of ridges and furrows defines the uniqueness of a fingerprint. Normally, ridge patterns are decomposed into local ridge characteristics, ridge bifurcation or ridge ending. These local ridge characteristics are generally known as minutiae points.

Reliable extraction of minutiae is one of the critical step in an automatic fingerprint identification system. A minutiae based fingerprint matching algorithm heavily relies on the quality of fingerprint images [1]. In poor quality fingerprint images noise destroy the ridge structure. Although, a human can identify true minutia of noisy image, but for computer (especially in online fingerprint identification) the minutiae points' detection is very difficult process. Thus, fingerprint image enhancement is believed to be the most important step in minutiae based fingerprint identification methods [3].

A general fingerprint enhancement approach composed of an initial estimation of local image parameters, followed by a contextual filtering stage, where local filters are adaptive to the local image characteristics. In last decade, nonlinear Partial Differential Equation (PDE) based diffusion has attracted much attention, because of its adaptive behaviour [4]. Nonlinear image diffusion was first introduced by Prona and Malik [5]. On the basis of their study, numbers of non-linear diffusion filters have been proposed $[6,7,8,9,10]$. Most of these techniques are based

DOI : 10.5121/ijma.2014.6203 
The International Journal of Multimedia \& Its Applications (IJMA) Vol.6, No.2, April 2014

on a scalar diffusivity which bound the diffusion flux always along the gradient direction. These techniques are linear and isotropic in nature and have same common disadvantages, like edge blurring. To overcome the short-come of this isotropic diffusion, anisotropic diffusion, which is nonlinear by nature, is proposed by Joachim Weickert [11] to keep oriented structure preserved. This technique is well known as Coherence Enhancement Diffusion (CED). In CED, the local structures of an image are used to diffuse the image, large coherence results large diffusion. Weickert proposes to modify the eigenvalues of diffusion matrix $\sum$, by setting

$$
\lambda_{\text {min }}=\alpha, \quad \lambda_{\text {max }}=\alpha+(1-\alpha) \exp \left(\frac{-1}{Q^{2}}\right)
$$

where $\alpha$ is a user defined parameter which is used to regulate the amount of smoothing. Although, CED can enhance coherent structure, it fails for some non-linear local structures. One of the reasons of its failure is that the eccentricity of diffusion is unbounded. Due to this, it gives larger value of eccentricity in high contrast region. To overcome this constrain, in this paper, we propose a Di- rectional Filter Bank (DFB) based robust orientation method to calculate reliable orientation estimation. By Using the robust orientation estimation we propose a method which automatically find the $\lambda_{\min }$ and $\lambda_{\max }$. From experiments, it is found that the proposed scheme not only preserve the global features but it also preserve the local feature under severe noise conditions, which Almansa algorithm failed to preserve.

The rest of the paper is organized as follow. Section II details the Coherence Enhancement Diffusion. In section III a new scheme for robust orientation estimation is proposed. Section IV describes how the pre-computed orientation is used in Coherence Enhancement Diffusion. In Section V testing and validation is presented and finally the paper is concluded in section VI.

\section{Coherence Enhanced Diffusion}

The diffusion process is thought to be equivalent to the process of energy minimizing variation:

$$
E(u)=\int_{\Omega}\left[\beta(u-f)^{2}+\operatorname{tr}\left(\Psi\left(\nabla u . \nabla u^{T}\right)\right)\right] d x d y
$$

Where $f$ is original image in domain $\Omega$ and $u$ is the diffused image.

is a small position. When diffusion matrix is identity, the diffusion equation reduced to Linear Scale-Space representation given by:

$$
\partial_{t} L=\frac{1}{2} \operatorname{div}(\nabla L)
$$

As fingerprint images have a very strong no isotropy, so we have to adapt the diffusion matrix to local image structure known as second moment matrix. The PDE of Nonlinear Tensor diffusion defined by [11] is given by:

$$
\partial_{t} L=\nabla \cdot(D \nabla L)
$$

where $\mathrm{D}$ is a diffusion tensor. For 2D images this diffusion tensor is then assumed to be: 
The International Journal of Multimedia \& Its Applications (IJMA) Vol.6, No.2, April 2014

$$
D=\left(\begin{array}{ll}
a & b \\
c & d
\end{array}\right)
$$

This 2 by 2 matrix always generate an ellipse. It contains $\lambda_{\min }, \lambda_{\max }$ and. Where $\lambda_{\min }$ the minor axis of the ellipse is, $\lambda_{\max }$ is its major axis and is the rotation angle. In general this ellipse is elongates in the direction orthogonal to the ridge. Coherence Enhancement Diffusion is a process that correctly use $\lambda_{\min }$ and $\lambda_{\max }$ according the underlying ridge structure. The diffusion tensor for nonlinear coherence enhancement can be of form:

$$
D=R^{T}\left(\begin{array}{ll}
c_{u} & 0 \\
0 & c_{v}
\end{array}\right) R
$$

where $R$ is a rotation matrix which describes the local coordinate system which is aligned along with the gradient vector observed at any scale u. Note that $c_{u}$ and $c_{v}$ can construct two one dimensional directional kernel: one scaling or performing diffusion along the $u$ direction and the other performing diffusion along $v$ direction. In other words we can say that by using appropriate values of $c_{u}$ and $c_{v}$ we can perform diffusion along the ridge direction or across the ridge direction. Normally $c_{u}$ is used to diffuse the noise along the ridge direction and $c_{v}$ for diffusion across the ridge direction.

The rotation matrix R given in equation 6 gives the eigenvectors of structure tensor. The structure tensor which implicitly calculates the angles of diffusion matrix is given by:

$$
S=\left(\begin{array}{ll}
s_{11} & s_{12} \\
s_{12} & s_{22}
\end{array}\right)=\left(\begin{array}{ll}
G_{x x}(x, y, \sigma) & G_{x y}(x, y, \sigma) \\
G_{x y}(x, y, \sigma) & G_{y y}(x, y, \sigma)
\end{array}\right)
$$

where $G_{x x}(x, y, \sigma), G_{x y}(x, y, \sigma)$ and $G_{y y}(x, y, \sigma)$ represents the Gaussian derivative filters. The Gaussian derivatives are mostly used for the scale space based vision applications. Analytically we can easily calculate the eigenvectors of the structure tensor and rotation matrix $R$. The diffusion tensor $D$ with components is given by:

$$
\begin{aligned}
& d_{11}=1 / 2\left(\left(c_{u}-c_{v}\right)+\frac{\left(c_{u}-c_{v}\right)-\left(s_{11}-s_{22}\right)}{\alpha}\right) \\
& d_{12}=\frac{\left(c_{u}-c_{v}\right) s_{12}}{\alpha} \\
& d_{22}=d_{11}=1 / 2\left(\left(c_{u}-c_{v}\right)-\frac{\left(c_{u}-c_{v}\right)-\left(s_{11}-s_{22}\right)}{\alpha}\right)
\end{aligned}
$$

Where

$$
\alpha=\sqrt{\left(s_{11}-s_{22}\right)^{2}+4 s_{12}^{2}}
$$

Therefore, the eigenvalues of structure tensor are given by: 
The International Journal of Multimedia \& Its Applications (IJMA) Vol.6, No.2, April 2014

$$
\begin{aligned}
& \lambda_{\text {min }}=1 / 2\left(s_{11}+s_{12}+\alpha\right) \\
& \lambda_{\text {max }}=1 / 2\left(s_{11}+s_{12}-\alpha\right)
\end{aligned}
$$

from these eigenvalues we can determine $c_{u}$ and $c_{v}$ as

$$
\begin{aligned}
& c_{u}=\max \left(0.01,1-e^{-\left(\lambda_{\min }-\lambda_{\max }\right)^{2}} / k^{2}\right) \\
& c_{v}=0.01
\end{aligned}
$$

\section{ROBUST ORIENTATION ESTIMATION}

Reliable orientation estimation is very useful for enhancing the quality of features to be detected in a given image. It can reduce the computation burden placed on post processing stage. In the presence of noise, it is very hard to get the reliable orientation estimation. In literature, different techniques have been used for orientation estimation e.g [12] uses a filter bank based approach, [13] uses waveform projection and spectral estimation is used by [14]. It is reported that these methods do not provide much accurate results, because they rely on fixed templates or filters. To manipulate coarse orientation estimation, mathematical modelling using Bayesain network [15] and complex polynomial are introduced. Later [16] introduces an enhanced gradient-based approach for reliable orientation estimation. In this scheme, dominant orientation of a base block from its four overlapping neighbourhoods is estimated and finally a best estimate is selected from least noise-affected neighbourhood. However, gradients are unreliable and error-prone on fingerprint images affected by noise like scares, smudges, dryness or wetness of finger [17].

[18] Uses directional filter bank (DFB) to extract the weak features of texture images. In DFB, the directional images contain linear features in a narrow directional band. It is found from [18] that these directional images contain significantly less noise as compared to the original image. However, fixed block/scale is used to compute the gradients for local orientation among directional images which restricts its capability for noise suppression. [19] propose a multiscale based DFB for orientation estimation. In this paper we proposed a new multiscale orientation estimation using Directional Filter Bank. We adopted the following steps.

Step I. The input image $L(x ; y)$ is decomposed into number of directional images $L(x ; y ; i)$ using Decimation-free Directional Filter bank [19], where $i=1,2, \ldots, n$ corresponding to quantized orientations associated with each directional image.

Step II. At a scale t, each directional image $L(x ; y ; i)$ is convolved by Gaussian Kernel as $L(x ; y$; $i ; t)=g(x ; y ; t) * L(x ; y ; i)$. This provide us with a scale-space representation.

Step III. For each scaled image L $(x ; y ; i ; t)$ the second moment matrix is computed as,

$$
\mu_{\text {simple }}=\left[\begin{array}{lc}
L_{x}^{2} & L_{x} L_{y} \\
L_{x} L_{y} & L_{y}^{2}
\end{array}\right]
$$

This $\mu_{\text {simple }}$ is further smoothed using a Gaussian Kernel having scale $\mathrm{S}$, where $\beta t^{2}$ and $\beta=2,3,4, \ldots, 8$ in our case 
The International Journal of Multimedia \& Its Applications (IJMA) Vol.6, No.2, April 2014

Step IV. For each $\mathrm{t}$, we got number of second moment matrix $\mu_{t, s}$ for each $\beta$ values. By using eigenvalue analysis, each $\mu_{t, s}$ is decomposed which provide us $\lambda_{\max }$ for each $\mu_{t, s} \mathrm{We}$ develop a measure similar to one suggested in [?], to pick one parameter $\mathrm{t}$ and $\mathrm{S}$ value as,

$$
A(x, y ; i)=t^{3 / 2}\left(\lambda_{\max }\right)^{2}
$$

Step V. For each directional image L(x; y; i) the energy measure is computed as,

$$
B(x, y ; i)=\max _{t, s} A(x, y ; i, t, S)
$$

Step VI. This new energy measure $B(x ; y ; i)$ associated with each directional image $L(x ; y ; i)$, reflects the strength of the orientations associated with a particular direction. Finally, we lineally combine all the quantized orientation using their respective strength measure $\theta_{i}$ and we got

$$
\theta(x, y)=\sum_{i=1}^{\omega} \frac{B(x, y ; i)}{\sum_{i=1}^{\omega} B(x, y ; i)} \theta_{i}
$$

\section{COHERENCE EnHANCEMENT Diffusion USING PRE-COMPUTED ORIENTATION FIELD}

The main difference of our proposed method to the other diffusion based methods, especially from [20], is that the diffusion tensor is fed with pre-computed orientation field. As discussed in section III, the proposed scheme for orientation estimation is more robust to noise and other degradations in the fingerprint than the gradient based methods. By feeding pre-computed orientation to diffusion can incredibly enhance low-quality fingerprints in particular. To embed the pre-computed orientation with diffusion tensor, tensor rotation in needed instead to simple vector multiplication. In tensor rotation, a rotation matrix is multiplied on left and a transpose of rotation matrix is multiplied on right of tensor matrix:

$$
\left(\begin{array}{ll}
D_{x x}^{\prime} & D_{x y}^{\prime} \\
D_{x y}^{\prime} & D_{y y}^{\prime}
\end{array}\right)=\left(\begin{array}{ll}
a & b \\
c & d
\end{array}\right)\left(\begin{array}{ll}
D_{x x} & D_{x y} \\
D_{x y} & D_{y y}
\end{array}\right)\left(\begin{array}{ll}
a & b \\
c & d
\end{array}\right)
$$

expansion of Eq. 16 yields.

$$
\begin{aligned}
& D_{x x}^{\prime}=a^{2} D_{x x}+c^{2} D_{y y}+2 a c D_{x y} \\
& D_{x y}^{\prime}=(a d+b c) D_{x y}+a b D_{x x}+c d D_{y y} \\
& D_{y y}^{\prime}=b^{2} D_{x x}+d^{2} D_{y y}+2 b d D_{x y}
\end{aligned}
$$




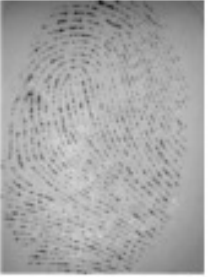

(a)

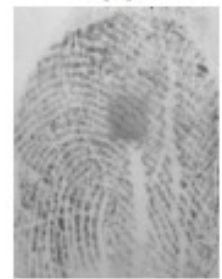

(d)

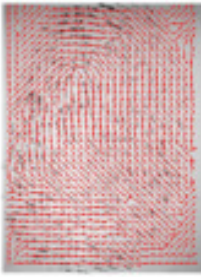

(b)

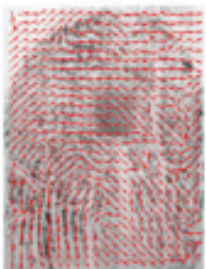

(e)

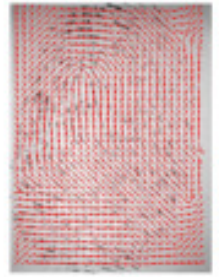

(c)

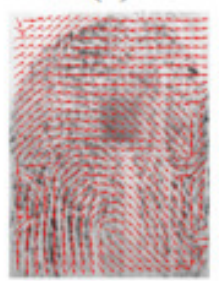

(f)

Figure 1: Real Fingerprint Images: The performance of [19] and the proposed is given. Two noisy images a) and d) from FVC database are used to test both the algorithms. b) and e) are the outputs of orientation estimation by [19] and c) an f) and the outputs of proposed method.

Let $D_{x x}^{\prime}=A, D_{x y}^{\prime}=B$ and $D_{y y}^{\prime}=C$. In order to rotate a reference frame through an angle $-\theta$, multiplication of $R(-\theta)$ on left and $R(-\theta)^{T}$ on right is required. This process can be visualized as rotating the ellipse through an angle $\theta=\theta_{f}$

$$
\left(\begin{array}{ll}
A & B \\
B & C
\end{array}\right)=\left(\begin{array}{cc}
\cos \theta & -\sin \theta \\
\sin \theta & \cos \theta
\end{array}\right)\left(\begin{array}{ll}
D_{x x} & D_{x y} \\
D_{x y} & D_{y y}
\end{array}\right)\left(\begin{array}{cc}
\cos \theta & -\sin \theta \\
\sin \theta & \cos \theta
\end{array}\right)
$$

expansion of Eq. 18 yields,

$$
\begin{aligned}
& A=\cos ^{2} \theta D_{x x}+\sin ^{2} \theta D_{y y}+2 \sin \theta \cos \theta D_{x y} \\
& B=\left(\cos ^{2} \theta+\sin ^{2} \theta\right) D_{x y}+a b D_{x x}+c d D_{y y} \\
& C=\sin ^{2} \theta D_{x x}+\cos ^{2} \theta D_{y y}+2 \sin \theta \cos \theta D_{x y}
\end{aligned}
$$

The diagonal angle can be calculated by setting $D_{x y}^{\prime}=0$. This implies,

$$
\begin{aligned}
& D_{x y}\left(\cos 2 \theta_{\text {diag }}\right)+\left(D_{x x}-D_{x y}\right) \sin 2 \theta=0 \\
& \tan 2 \theta_{\text {diag }}=2 D_{x y} /\left(D_{x x}-D_{x y}\right) \\
& \text { so, } \\
& \theta_{\text {diag }}=\arctan \left[2 D_{x y} /\left(D_{x x}-D_{x y}\right)\right] / 2
\end{aligned}
$$


The International Journal of Multimedia \& Its Applications (IJMA) Vol.6, No.2, April 2014

\subsection{Calculating Robust Eigenvalues}

In order to compute more robust eigenvalues by using pre-computed orientation, we used the hessian matrix of directional image $I 1$ in new coordinates, given by [18], as,

$$
H^{\prime}=\left(\begin{array}{ll}
h_{11} & h_{12} \\
h_{21} & h_{22}
\end{array}\right)=\left(\begin{array}{ll}
\frac{\partial^{2} I_{1}}{\partial x^{\prime 2}} & \frac{\partial^{2} I_{1}}{\partial x^{\prime} \partial y^{\prime}} \\
\frac{\partial^{2} I_{1}}{\partial x^{\prime} \partial y^{\prime}} & \frac{\partial^{2} I_{1}}{\partial y^{\prime 2}}
\end{array}\right)
$$

Where,

$$
\begin{aligned}
& \frac{\partial^{2} I_{1}}{\partial x^{\prime 2}}=\frac{\partial^{2} I_{1}}{\partial x^{2}} \cos ^{2} \theta_{i}+\frac{\partial^{2} I_{1}}{\partial x \partial y} \sin \left(2 \theta_{i}\right)+\frac{\partial^{2} I_{1}}{\partial y^{2}} \operatorname{sins}^{2} \theta_{i} \\
& \frac{\partial^{2} I_{1}}{\partial y^{\prime 2}}=\frac{\partial^{2} I_{1}}{\partial x^{2}} \sin ^{2} \theta_{i}-\frac{\partial^{2} I_{1}}{\partial x \partial y} \sin \left(2 \theta_{i}\right)+\frac{\partial^{2} I_{1}}{\partial y^{2}} \cos ^{2} \theta_{i} \\
& \frac{\partial^{2} I_{1}}{\partial x^{\prime} \partial y^{\prime}}=-\frac{1}{2} \frac{\partial^{2} I_{1}}{\partial x^{2}} \sin \left(2 \theta_{i}\right)+\frac{\partial^{2} I_{1}}{\partial x \partial y} \cos \left(2 \theta_{i}\right)+\frac{1}{2} \frac{\partial^{2} I_{1}}{\partial y^{2}} \sin \left(2 \theta_{i}\right)
\end{aligned}
$$

In [18] $h_{11}$ is defined as $\lambda_{1}$ and $h_{22}$ as $\lambda_{2}$. In order to get better results in noisy condition $\frac{\partial^{2} I_{1}}{\partial x^{\prime 2}}$ is replaced by $A, \frac{\partial^{2} I_{1}}{\partial x^{\prime} \partial y^{\prime}}$ by $B$ and $\frac{\partial^{2} I_{1}}{\partial y^{\prime 2}} C$, which yields,

$$
\begin{aligned}
& \lambda_{1-r}=A \cos ^{2} \theta_{i}+B \sin \left(2 \theta_{i}\right)+C \sin ^{2} \theta_{i} \\
& \lambda_{2-r}=A \sin ^{2} \theta_{i}-B \sin \left(2 \theta_{i}\right)+C \cos ^{2} \theta_{i}
\end{aligned}
$$

\subsection{FinAL DifFUSION TENSOR}

For final diffusion tensor matrix, we use,

$$
\left(\begin{array}{ll}
A_{f} & B_{f} \\
B_{f} & C_{f}
\end{array}\right)=\left(\begin{array}{cc}
\cos \theta & -\sin \theta \\
\sin \theta & \cos \theta
\end{array}\right)\left(\begin{array}{ll}
\lambda_{1-r} & 0 \\
0 & \lambda_{2-r}
\end{array}\right)\left(\begin{array}{cc}
\cos \theta & -\sin \theta \\
\sin \theta & \cos \theta
\end{array}\right)
$$

Let $I_{1} x=\cos \theta, I_{1} y=\sin \theta, I_{2} x=-\sin \theta$ and $I_{1} y=\cos \theta$. By solving above matrix, we get

$$
\begin{aligned}
& A_{f}=\lambda_{1-r} I_{1} x^{2}+\lambda_{2-r} I_{2} x^{2} \\
& B_{f}=\lambda_{1-r} I_{1} x I_{1} y+\lambda_{2-r} I_{2} x I_{2} y \\
& C_{f}=\lambda_{1-r} I_{1} y^{2}+\lambda_{2-r} I_{2} y^{2}
\end{aligned}
$$

\section{TESTING AND VALIDATION}

Statistically, the set of minutiae denoted by symbol $M$ were algorithmically obtained and evaluated against the set of minutiae marked by an expert denoted by symbol $F$ and the minutiae are classified into two types: termination and bifurcation. Each minutiae point $m$ compartmentalized into one of the following classes: 
The International Journal of Multimedia \& Its Applications (IJMA) Vol.6, No.2, April 2014

- Correct: The correlation between minutiae points generated through the algorithm and those obtained from the expert is perfect position, i.e $\left(m \in F \cap M\right.$ and type $_{F}(m)=$ type $\left._{M}(m)\right)$

- False: The expert did not mark the location corresponding to the one obtained through the algorithm, i.e $(m \in M, m \notin F)$

- Missing: The minutiae location has been marked by the expert but couldn't be seen within the algorithmically generated image, i.e $(m \notin M, m \in F)$

- Misclassified: The minutiae location marked by the expert could be is also the one found by the method with the exception that one has been classified as bifurcation and other has been classified as termination, i.e $\left(m \in F \cap M\right.$ and type $_{F}(m) \neq$ type $\left._{M}(m)\right)$

Compartmentalization of all the minutiae set is followed by the performance measurement which is computed statistically. This is done on minutiae obtained from the algorithm as well as minutiae marked by the experts which makes the performance measurement relative to either total number of minutiae found algorithmically or the total number of minutiae found by the expert markings. The measures relative to minutiae marked by the expert which detects the aptitude of the technique to detect correct minutiae are as follows:

- Missing minutiae is defined as $\frac{M i s \sin g}{|F|}$

- False minutiae is defined as $\frac{\text { False }}{|F|}$

- Misclassified minutiae is defined as $\frac{\text { Missclassified }}{|F|}$

- Correct minutiae is defined as $\frac{\text { Correct }}{|F|}$

- Total error which is the summation of false positive, false negative and misclassified errors is defined as $\frac{\text { Mis } \sin g \cup \text { False } \cup \text { Missclassified }}{|F|}$

- Classification rate for the purpose of enhancing the graphical visualization is defined as $\frac{\text { Correct }}{\text { Missclassified }}$

Large number of missing minutiae could be tolerated to certain extent but the large number of false minutiae would lead to larger noise rate within the mapping stage. On the other hand, prominence of classification error is based on the manner in which the mapping algorithm deals with the types of minutiae. Using the techniques described above, the database of true minutiae was generated, by using the sample images given in Fig. 6 which is used to evaluate the 
The International Journal of Multimedia \& Its Applications (IJMA) Vol.6, No.2, April 2014

orientation and scale-matched filters to produce improved input image along with the techniques described above.
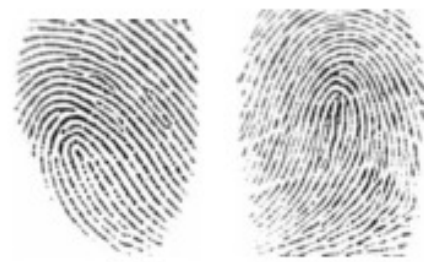

(Image 1)

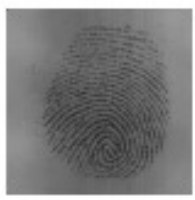

(Image5)

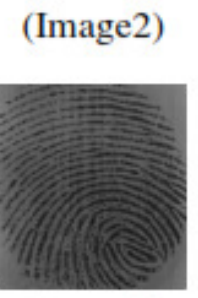

(Image6)
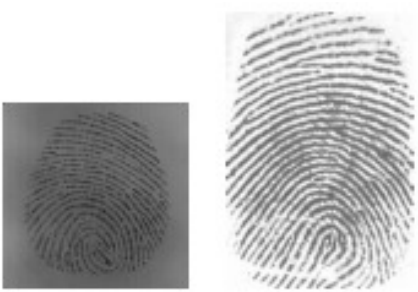

(Image3)

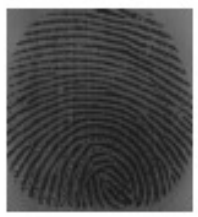

(Image7)
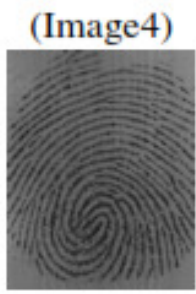

(Image8)

Fig. 2 sample images used for evaluation measure

The table compares the result obtained using the above method.

\begin{tabular}{|l|l|l|l|l|}
\hline Proposed & Correct & False & Missing & Misclassified \\
\hline Image1 & 10 & 5 & 5 & 4 \\
\hline Image2 & 9 & 5 & 5 & 9 \\
\hline Image3 & 20 & 3 & 4 & 7 \\
\hline Image4 & 13 & 2 & 5 & 5 \\
\hline Image5 & 19 & 10 & 4 & 6 \\
\hline Image6 & 22 & 4 & 8 & 9 \\
\hline Image7 & 12 & 4 & 5 & 16 \\
\hline Image8 & 23 & 4 & 6 & 8 \\
\hline Mean & 17.25 & 5.12 & 4.75 & 8 \\
\hline
\end{tabular}

Table1. This table shows the evaluation measure comparing minutiae of proposed algorithm 
The International Journal of Multimedia \& Its Applications (IJMA) Vol.6, No.2, April 2014

\begin{tabular}{|l|l|l|l|l|}
\hline$[12]$ CED & Correct & False & Missing & Misclassified \\
\hline Image1 & 14 & 2 & 4 & 2 \\
\hline Image2 & 5 & 4 & 2 & 15 \\
\hline Image3 & 8 & 3 & 4 & 20 \\
\hline Image4 & 1 & 4 & 2 & 5 \\
\hline Image5 & 3 & 2 & 4 & 22 \\
\hline Image6 & 16 & 7 & 3 & 20 \\
\hline Image7 & 4 & 2 & 2 & 30 \\
\hline Image8 & 16 & 3 & 6 & 7 \\
\hline Mean & 9.875 & 3.375 & 3.5 & 15.12 \\
\hline
\end{tabular}

Table2. This table shows the evaluation measure comparing minutiae of [11] CED Enhancement algorithm

The average number of minutiae points present in the image marked by the expert and the ones marked by the algorithm for all the eight images was taken, which represented $F$ This was computed to be 15 using which some performance measures were computed. For the proposed method, classification error which is calculated as misclassified over correctly detected minutiae was found to be

$$
\frac{\text { Missclassified } / F \cap M}{|F|} * 100=1.8 \%
$$

For the CED method, classification error which is calculated as misclassified over correctly detected minutiae was found to be

$$
\frac{\text { Missclassified } / F \cap M}{|F|} * 100=2.36 \%
$$

In Fig3, the bar chart shows the performance evaluation measure of both schemes.

\section{Conclusions}

Coherence enhancement diffusion filters are the one which have control on the size/ shape of the filter but, this use derivative for rotation matrix which is not considered as a reliable method. Therefore, in this work presents a new and a reliable method for robust orientation estimation is proposed to explicitly calculate the orientation field of a fingerprint image. By using this robust orientation new local scales for Diffusion matrix are adaptively calculated. The experiments conducted on the noisy images showed promising results of the proposed scheme. 


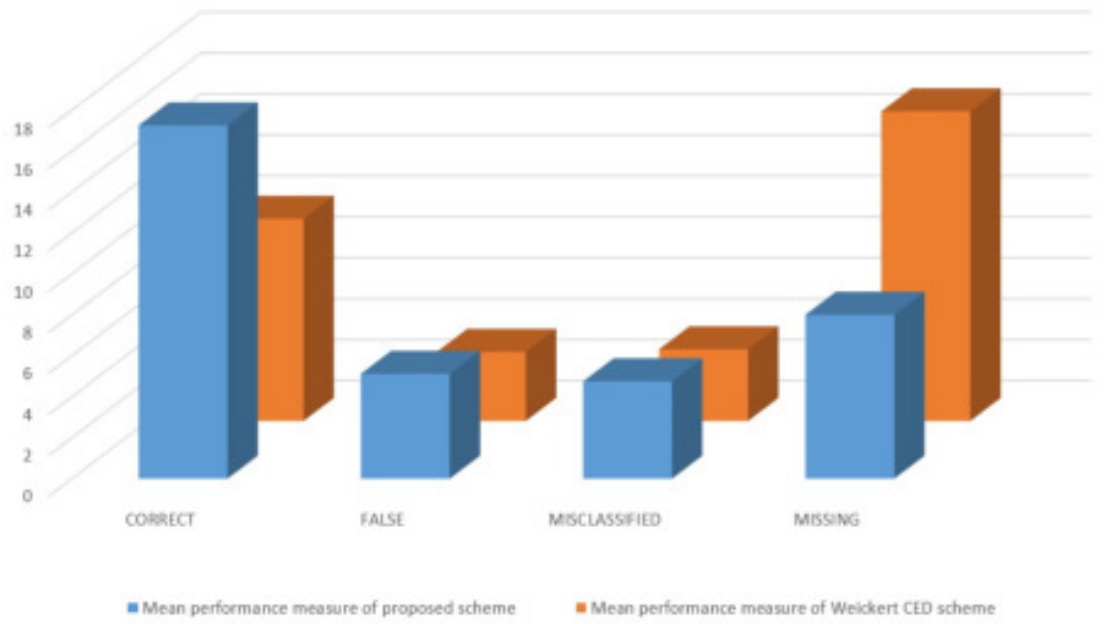

Fig. 3. Performance Evaluation Measure of both schemes

\section{REFERENCES}

[1] L. Hong, Y. Wan, and A. Jain, "Fingerprint image enhancement: Algorithm and performance evaluation," IEEE Transactions on Pattern Analysis and Machine Intelligence, vol. 20, pp. 777789, 1998.

[2] M. A. Khan amd A. Khan, T. Mahmmod, M. Abbas, and N. Muhammad, "Fingerprint image enhancement using principal component analysis (pca) filters," in International Conference on Information and Emerging Technologies (ICIET), 2010.

[3] M.A.U. Khan, T. M. Khan, and S. A. Khan, "Coherence enhancement dif- fusion using multi-scale dfb," in 7th International Conference on Emerging Technologies, 2011.

[4] J Weickert, "A review of nonlinear diffusion filtering," cale-Space Theory in Computer Vision, vol. 1252, pp. 3-28, 1997. [5] J Weickert, "A review of nonlinear diffusion filtering," cale-Space Theory in Computer Vision, vol. 1252, pp. 3-28, 1997.

[5] P Perona and J Malik, "Scale-space and edge detection using anisotropic diffusion.," IEEE Trans Mach Intell., vol. 12(7), pp. 629639, 1990.

[6] L Alvarez, P-L Lions, and J-M Morel, "Image selective smoothing and edge detection by nonlinear diffusion,” II SIAM J Numer Anal, vol. 29, pp. 845866, 1992.

[7] G Sapiro, "Tannenbaum, affine invariant scale-space," International Journal of Computer Vision, vol. 11, pp. 25-44, 1993.

[8] L Rudin, S Osher, and E Fatemi, "Nonlinear total variation based noise removal algorithms," Modeliz Mat Traitement d Images, p. 149179, 1992.

[9] HM Salinas and D Cabrera Fernandez, "Comparison of pde-based nonlinear diffusion approaches for image enhancement and denoising in optical coherence tomography," IEEE Transactions on Medical Imaging, vol. 26, pp. 761-771, 2007.

[10] D Fang, Z Nanning, and X Jianru, "Image smoothing and sharpening based on nonlinear diffusion equation.," Signal Processsing, vol. 88(11), pp. 28502855, 2008.

[11] J Weickert, "Coherence-enhancing diffusion filtering," Int. J. Computer Vision,, vol. 31, pp. 111-127, 1999. 
The International Journal of Multimedia \& Its Applications (IJMA) Vol.6, No.2, April 2014

[12] A.K. Jain, S. Pankanti, and L. Hong, "A multichannel approach to finger- print classification," IEEE Trans. Pattern Anal. Machine Intell, vol. 21, pp. 348359, 1999..

[13] ] B.G. Sherlock, D.M. Monro, and K. Millard, "Fingerprint enhancement by directional fourier filtering,” IEE Proc. Vision, Image Signal Process., vol. 141, pp. 8794., 1994.

[14] C.L. Wilson, G.T. Candela, and C.I. Watson, "Neural network fingerprint classification," J. Artif. Neural Networks, vol. 1, pp. 203228, 1994.

[15] S.C. Dass, "Markov random field models for directional field and singularity extraction in figerprint images,” IEEE Trans. Image Process, vol. 13, pp. 13581367, 2004.

[16] Yi Wang, Jiankun Hu, and Fengling Han, "Enhanced gradient-based algo- rithm for the estimation of fingerprint orientation fields," Applied Mathe- matics and Computation, vol. 185, pp. 823833, 2007.

[17] Gottschlich C, P. Mihailescu, and A. Munk, "Robust orientation field es- timation and extrapolation using semilocal line sensors,” IEEE Trans. Inf. Forensics Sec., vol. 4, pp. 802811, 2009.

[18] P. T. H. Truc, M. A. U. Khan, Y. K. Lee, and T. S. Kim, "Vessel enhancement filter using directional filter bank," Computer Vision and Image Understand- ing, vol. 113, pp. 101-112, 2009.

[19] A.K. Jain, S. Pankanti, and L. Hong, “A multichannel approach to fingerprint classification,” IEEE Trans. Pattern Anal. Machine Intell, vol. 21, pp. 348359, 1999.

[20] Muhammad A. K. Khan and Wadee Alhalabi, "Robust multi-scale orienta- tion estimation: Spatial domain vs fourier domain," in International Confer- ence on Communications, Signal Processing, and their Applications, 2013.

[21] Andres Almansa and Tony LIndeberg, "Fingerprint enhancement by shape adaption of scale)space operators with automatic scale selection," IEEE transaction on image processing, vol. 9, pp. 20272041, 2000.

\section{AUTHORS}

Dr Yinan Kong

Dr Kong leads the VLSI Research Group at Macquarie. He is also currently the Director of Higher Degree Research, Department of Engineering, Macquarie University, Sydney, Australia. Dr Kong obtained his PhD from the University of Adelaide, Australia. During his research at the VLSI Research Group at Macquarie University and Centre for High Performance Integrated Technologies and Systems (CHiPTec) at the University of Adelaide, Dr Kong focused on digital VLSI design at an arithmetic level for specific applications like cryptography and DSP. He achieved the hardware construction of a 1024-bit RSA cryptosystem at only $0.4 \mathrm{~ms}$ per decryption, the fastest ever FPGA implementation of RSA. In the meantime, he developed Bachelor Degree of Computer Engineering at Macquarie Engineering as well as postgraduate teaching in embedded computer system design and computer arithmetic with hardware focused.

Tariq Mahmood Khan

Tariq Mahmmod Khan is interested in both Digital Image Processing (with an emphasis on biometrics) and VLSI. He is currently involved in the hardware implementation of multi-modal biometrics project, led by Yinan Kong. The main goal of this project is to develop a fast processor for different design approaches. The aim is to test these approaches on a multi-modal biometric system consisting fingerprint, iris and face. Prior to starting his $\mathrm{PhD}$ in Engineering at Macquarie University, he received a degree in BS Computer Engineering from COMSATS Institute of Information \& Technology, Islamabad Pakistan and MSc in Computer Engineering from University

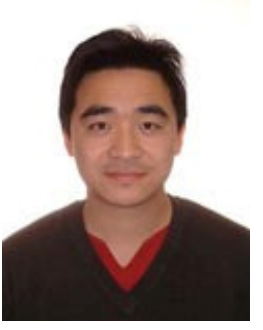
of Engineering \& Technology, Taxila Pakistan. 